\title{
Global persistence exponent of the two-dimensional Blume-Capel model
}

\author{
Roberto da Silva*, Nelson A. Alves ${ }^{\dagger}$, and J. R. Drugowich de Felício \\ Departamento de Física e Matemática, FFCLRP Universidade de São Paulo, Avenida Bandeirantes 3900 , \\ CEP 014040-901, Ribeirão Preto, São Paulo, Brazil
}

(May, 14, 2002)

\begin{abstract}
The global persistence exponent $\theta_{g}$ is calculated for the two-dimensional Blume-Capel model following a quench to the critical point from both disordered states and such with small initial magnetizations. Estimates are obtained for the nonequilibrium critical dynamics on the critical line and at the tricritical point. Ising-like universality is observed along the critical line and a different value $\theta_{g}=1.080(4)$ is found at the tricritical point.
\end{abstract}

Keywords: persistence, dynamic exponent, critical phenomena, Blume-Capel model, Monte Carlo simulation.

PACS: 64.60.Ht, 05.70.Ln, 05.50.+q

Recent advances in nonequilibrium physics have enlarged our understanding of critical phenomena. As shown by Janssen et al. [1] and Huse [2], universality and scaling behavior are already present in systems since the early stages of their relaxation processes after quenching from high temperatures to the critical one. This evolution is governed by a new independent exponent $\theta$. Systems characterized by a nonconserved order parameter, model $\mathrm{A}$ in the terminology of Halperin et al. [3], are described by a scaling function soon after a microscopic time scale $t_{m i c}$. Its general form for the $k$ th moment of the magnetization (e.g., in a ferromagnet) reads as

$$
M^{(k)}\left(t, \tau, L, m_{0}\right)=b^{-k \beta / \nu} M^{(k)}\left(b^{-z} t, b^{1 / \nu} \tau, b^{-1} L, b^{x_{0}} m_{0}\right) .
$$

Here $b$ is an arbitrary spatial scaling factor, $t$ is the time evolution and $\tau$ is the reduced temperature, $\tau=\left(T-T_{c}\right) / T_{c}$. As usual, the exponents $\beta$ and $\nu$ are the well-known static exponents, whereas $z$ is the dynamic one. Equation (1) depends on the initial magnetization $m_{0}$ and gives origin to the new exponent $x_{0}$, scaling dimension of the initial magnetization, related to $\theta$ by $x_{0}=\theta z+\beta / \nu$.

The nonequilibrium short-time exponent $\theta$ can be obtained at the critical temperature from the scaling form of the first moment $[1,4,5], M\left(t, m_{0}\right) \sim m_{0} t^{\theta}$. Usually, this exponent assumes a positive value corresponding to a critical initial slip, which is related to the anomalous behavior of the magnetization when the system is quenched to $T_{c}$. Numerical works indicate negative values for $\theta$ at the tricritical point [6] of the $S=1$ Blume-Capel model [7-9] in two dimensions $(2 d)$ as well as for the Baxter-Wu [10], Ising model with three-spin interactions [11] and for the four-state Potts model [12]. The indication of a negative value for this exponent was theoretically deduced by Janssen and Oerding [13] from a study of non-equilibrium relaxation at a tricritical point. Numerical simulations of the $S=1$ Blume-Capel model [6] present for the dynamical exponents the values $\theta=-0.53(2)$ and $z=2.21(2)$ at the tricritical point and values compatible with heat-bath dynamics for the two-dimensional Ising model $(\theta=0.191(1)$ $[4,14], z=2.156(2)[15])$ on the critical line.

Under the same nonequilibrium conditions, a second critical exponent has been presented in the literature [16]: the global persistence exponent $\theta_{g}$. It is related to the probability $P(t)$ that the global order parameter has not changed sign up to time $t$ after a quench to $T_{c}, P(t) \sim t^{-\theta_{g}}$. This exponent has emerged from the concept of the local persistence phenomena in coarsening dynamics at $T=0$ [17-22]. As argued by Majumdar et al. [16], if the dynamics of the global order parameter is described by a Markovian process, $\theta_{g}$ is not a new independent exponent and it can be related to other critical exponents,

$$
\theta_{g} z=\lambda-d+1-\eta / 2 .
$$

Here $\lambda$ is the nonequilibrium exponent of the auto-correlation function $[1,2]$,

$$
A(t)=\frac{1}{L^{d}}\left\langle\sum_{i} S_{i}(0) S_{i}(t)\right\rangle \sim t^{-\lambda / z},
$$

\footnotetext{
*E-mail: rsilva@dfm.ffclrp.usp.br

${ }^{\dagger}$ E-mail: alves@quark.ffclrp.usp.br

${ }^{\ddagger}$ E-mail: drugo@usp.br
} 
which is related to the short-time exponent, $\lambda=d-\theta z$. Therefore, Eq. (2) can be rewritten as

$$
\theta_{g} z=-\theta z+\frac{d}{2}-\frac{\beta}{\nu}
$$

However, the time evolution of the order parameter is, in general, a non-Markovian process and $\theta_{g}$ turns out to be a new independent critical exponent describing the stochastic dynamic process toward the equilibrium.

Contrary to the local persistence, the global persistence has been less studied. Results have only been reported for the $n \rightarrow \infty$ limit of the $O(n)$ model $\left(\theta_{g}=(d-2) / 4\right.$ for $2<d<4$ or $\theta_{g}=1 / 2$ for $\left.d>4\right)$ and to order $\epsilon=4-d$ near $d=4\left(\theta_{g}=1 / 2-\epsilon(n+2) /(4 n+32)+O\left(\epsilon^{2}\right)\right)$, for $d=1$ Ising model $\left(\theta_{g}=1 / 4\right)$ [16], and $d=2$ Ising model [16,23,24], as shown in Table I. As remarked in [16], relation (2) is satisfied for $n=\infty$ limit of the $O(n)$ model, to first order in $\epsilon=4-d$, and also for $d=1$ Ising model. However, for the $O(n)$ model, it has been shown [25] that the scaling relation (2) for Markovian process is violated at order $\epsilon^{2}$.

In this paper we investigate the universality aspects of the global persistence exponent for the Blume-Capel model. This exponent is obtained by the straight application of the power law behavior $P(t) \sim t^{-\theta_{g}}$ and by means of time series data collapse. This study is also performed under different initial conditions: random choices of all spins and sharp preparations of samples with defined and nonzero magnetizations $m_{0}$ [24].

The Blume-Capel [7] (BC) model is a spin-1 model which has been used to describe the behavior of ${ }^{3} \mathrm{He}-{ }^{4} \mathrm{He}$ mixtures along the $\lambda$ line and near the critical mixing point. Apart from its practical interest, the BC model has intrinsic interest since it is the simplest generalization of the Ising model $(s=1 / 2)$ exhibiting a rich phase diagram with first and second-order transition lines and a tricritical point. The Hamiltonian of the two-dimensional model is

$$
H=-J \sum_{<i, j>} S_{i} S_{j}+D \sum_{i=1} S_{i}^{2}
$$

where $\langle i, j\rangle$ indicates nearest neighbors on $L^{2}$ lattices and $S_{i}=\{-1,0,1\}$. The parameter $J$ is the exchange coupling constant and $D$ is the crystal field. We remark that along the critical line, this model presents a critical behavior similar to the Ising model. However, exactly at the tricritical point the exponents change abruptly. In [26] finite-size scaling combined with conformal invariance permitted to observe a smooth change between Ising-like and tricritical behavior. Ising-like behavior is reached only when $L \rightarrow \infty$, leading to the exact values of the Ising model critical exponents.

In order to evaluate the persistence probability $P(t)$, we define $\rho(t)$ as the fraction of samples which change their signals for the first time at the instant $t$. Our probability $P(t)$ is numerically calculated from the cumulative distribution function such that the total magnetization does not cross the origin up to time $t$,

$$
P(t)=1-\sum_{t^{\prime}=1}^{t} \rho\left(t^{\prime}\right) .
$$

The spins $\left\{S_{i}\right\}$ are updated by the heat-bath algorithm and our statistics rely on $N_{B}=5$ independent bins with $N_{S}=40000$ samples for $t$ up to $1000 \mathrm{MC}$ sweeps and lattice size $L=80$. We quote estimates for the time intervals $\left[t_{i}, t_{f}\right]$ with the highest values of goodness-of-fit $Q$ [27] for the linear regression obtained at every 10th measurement.

Table II lists the points on the second order critical line and the tricritical point where we have performed our simulations. This table presents estimates for $\theta_{g}$ in function of different magnetizations $m_{0}$ to explore the effect of the initial configurations on the behavior of $P(t)$. Linear extrapolations for $m_{0} \rightarrow 0$ are presented in the last column. Here we follow the sharp preparation technique to set the value $m_{0}$. Our typical time intervals for $m_{0}=0.0050$ correspond to $[100,500]$ (critical points) and $[40,400]$ (tricritical point). Different time intervals with accepted values for $Q$, present compatible results within our error estimates.

Figures 1 and 2 illustrate, respectively, the decay of persistence probability for the specific critical point $D / J=0$, $k_{B} T / J=1.6950$ and for the tricritical point.

Our simulations of the BC model on the critical line reproduce (see Table II) the estimates obtained by Schulke and Zheng [24] for the $2 d$ Ising model with $m_{0}=0.0005: \theta_{g}=0.238(3)$ (with the HB dynamics) and $\theta_{g}=0.236(3)$ (Metropolis algorithm). On the other hand, our simulations present the largest deviation (compared with the Ising model exponent) for the largest initial magnetization $m_{0}=0.0050$, but the expected universality is recovered as $m_{0} \rightarrow 0$. It becomes clear the importance of the initial configurations in measuring the persistence exponent.

At the tricritical point we observe a faster decay of the persistence probability $\left(\theta_{g}=1.080(4)\right)$, characterizing a different persistence behavior. We repeat our simulations for two new critical points [28] $D / J=1.87, k_{B} T / J=0.800$ and $D / J=1.95, k_{B} T / J=0.650$, closer to the tricritical one to follow this cross over effect. In this case, the 
persistence probability can be fitted by a power law function only for short time intervals. Hence, we have to restrict our analysis to shorter time intervals in order to obtain acceptable values for the goodness-of-fit. We obtain for the first point $\theta_{g}=0.172(3)$ in time interval [80,400], while for the second point, closer to the tricritical one, we have to restrict further the interval to $[100,300]$, leading to the estimate $\theta_{g}=0.345(5)$. Hence, this very different behavior characterized by those new values of $\theta$ indicates a 'transition' from the Ising-like $\theta_{g} \sim 0.23$ to the tricritical $\theta_{g} \sim 1.08$ for finite lattices.

The initial magnetization dependence of $P(t)$ can be cast in the following finite-size scaling relation [16],

$$
P(t)=t^{-\theta_{g}} f\left(t / L^{z}\right)=L^{-\theta_{g} z} \tilde{f}\left(t / L^{z}\right)
$$

which renders a different method to obtain the exponent $\theta_{g}$ from lattice sizes $L_{1}$ and $L_{2}$ [16]. For this end we define $W(t, L)=L^{\theta_{g} z} P(t)$, which turns out to be a function of $t / L^{z}$. Therefore, if we fix the dynamic exponent $z$, the exponent $\theta_{g}$ can be obtained by collapsing the time series $W\left(t_{2}, L_{2}\right)=f\left(t_{2} / L_{2}^{z}\right)$ onto $W\left(t_{1}, L_{1}\right)=f\left(t_{1} / L_{1}^{z}\right)$ as follows. Under re-scaling, with $b=L_{2} / L_{1},\left(L_{2}>L_{1}\right)$, we obtain

$$
W\left(t_{2}, L_{2}\right)=\widetilde{W}\left(b^{z} t_{1}, b L_{1}\right),
$$

and the best estimate for $\theta_{g}$ corresponds to the minimization of

$$
\chi^{2}\left(\theta_{g}\right)=\sum_{t}\left(\frac{W(t, L)-\widetilde{W}\left(b^{z} t, b L\right)}{|W(t, L)|+\left|\widetilde{W}\left(b^{z} t, b L\right)\right|}\right)^{2}
$$

by interpolating $\widetilde{W}$ to the time values $b^{z} t$.

This method is applied to the critical point $D / J=0, k_{B} T / J=1.6950$ and to the tricritical one. We performed simulations with lattice sizes $L=10,20,40$ and 80 and initial disordered states to study the finite size dependence of $\theta_{g}$. Our simulations also rely on $N_{s}=40000$ samples and $N_{b}=5$ bins. The collapse obtained from our largest pairs of lattices $\left(L_{1}, L_{2}\right)=(40,80)$ is displayed in Fig. 3 for the tricritical point. A similar figure (not shown) is also obtained for the critical one. Results for the persistence exponent are presented in Table III with the input values $z=2.106$ and $z=2.215$, respectively for the critical and tricritical point [6]. Here, we can observe a good agreement between both methods. However, we remark that the collapse method gives less precise results.

Now, we return to Eq. (4) to analyze the markovian aspects of the time evolution of the magnetization. We verify that our numerical precision for both the exponents [6] $\theta$ and $\theta_{g}$, evaluated on the critical line and at the tricritical point, allows us to detect the non-markovian behavior. Thus, these results substantiate the independence of global persistence and short-time exponents, characteristic of a non-markovian dynamic evolution of the magnetization.

In summary, we have studied the effects of the initial magnetization on $P(t)$ and evaluated the global persistence exponent of the Blume-Capel model from different methods (linear extrapolation $m_{0} \rightarrow 0$ and collapse). The universality and independence of this dynamic exponent is explicitly shown for the BC model along the critical line. In addition, the power law time dependence of the persistence probability is also exhibitted at the tricritical point, presenting a faster decay when compared with the Ising model exponent.

R. da Silva and J. R. Drugowich de Felício gratefully acknowledge support by FAPESP and N.A. Alves by CNPq (Brazil).

[1] H. K. Janssen, B. Schaub and B. Schmittmann, Z. Phys. B 73, 539 (1989).

[2] D. A. Huse, Phys. Rev. B 40, 304 (1989).

[3] B. I. Halperin, P. C. Hohenberg and S-K. Ma, Phys. Rev. B 10, 139 (1974).

[4] K. Okano, L. Schülke, K. Yamagishi and B. Zheng, Nucl. Phys. B 485 [FS], 727 (1997).

[5] B. Zheng, Int. J. Mod. Phys. B 12, 1419 (1998).

[6] R. da Silva, N.A. Alves and J.R. Drugowich de Felício, Phys. Rev. E 66, 026130 (2002).

[7] M. Blume, Phys. Rev. 141, 517 (1966). H. W. Capel, Physica 32, 966 (1966); 33, 295 (1967); 37, 423 (1967). 
[8] M. Blume, V. J. Emery and R. B. Griffiths, Phys. Rev. A 4, 1071 (1971).

[9] I. D. Lawrie and S. Sarbach, in Phase Transitions and Critical Phenomena, vol.9. Edited by C. Domb and J.L. Lebowitz, 1984 (Academic Press).

[10] E. Arashiro and J.R. Drugowich de Felício (to appear).

[11] C.S. Simões and J.R. Drugowich de Felício, Mod. Phys. Lett. B 15, 487 (2001).

[12] R. da Silva and J.R. Drugowich de Felício (to appear).

[13] H. K. Janssen and K. Oerding, J. Phys. A: Math. Gen. 27, 715 (1994).

[14] P. Grassberger, Physica A 214, 547 (1995).

[15] R. da Silva, N.A. Alves and J.R. Drugowich de Felício, Phys. Lett. A 298, 325 (2002).

[16] S.N. Majumdar, A.J. Bray, S.J. Cornell and C. Sire, Phys. Rev. Lett. 77, 3704 (1996).

[17] B. Derrida, A.J. Bray and C. Godrèche, J. Phys. A 27, L357 (1994).

[18] A.J. Bray, B. Derrida and C. Godrèche, Europhys. Lett. 27, 175 (1994).

[19] D. Stauffer, J. Phys. A27, 5029 (1994).

[20] B. Derrida, V. Hakim, V. Pasquier, Phys. Rev. Lett. 75, 751 (1995).

[21] S.N. Majumdar and C. Sire, Phys. Rev. Lett. 77, 1420 (1996).

[22] S.N. Majumdar, C. Sire, A.J. Bray, and S.J. Cornell, Phys. Rev. Lett. 77, 2867 (1996).

[23] D. Stauffer, Int. J. Mod. Phys. C7, 753 (1996).

[24] L. Schülke and B. Zheng, Phys. Lett. A 233, 93 (1997).

[25] K. Oerding, S. J. Cornell, and A.J. Bray, Phys. Rev. E 56, R25 (1997).

[26] F. C. Alcaraz, J. R. Drugowich de Felício, R. Köberle and J. F. Stilck, Phys. Rev. B 32, 7469 (1985).

[27] W. Press et al., Numerical Recipes (Cambridge University Press, London, 1986).

[28] P.D. Beale, Phys. Rev. B 33, 1717 (1986). 
TABLE 1. The global persistence exponent $\theta_{g}$ for the $2 d$ Ising model with random and with pre-fixed small initial magnetization $m_{0}$.

\begin{tabular}{clcc}
\hline \hline & & & \\
Reference & random & $m_{0}=0.0010$ & $m_{0}=0.0005$ \\
\hline$[24]$ & $0.233(5)$ & $0.237(5)$ & $0.238(3)$ \\
{$[23]$} & $0.225(10)$ & & \\
{$[16]$} & $0.233(9)$ & & \\
\hline \hline
\end{tabular}

TABLE 2. The global persistence exponent $\theta_{g}$ from the power law behavior for the $2 d$ Blume-Capel model for different initial magnetizations $m_{0}$. Last column contains our linear extrapolation to $m_{0}=0$.

\begin{tabular}{|c|c|c|c|c|c|}
\hline $\mathrm{D} / \mathrm{J}$ & $k_{B} T / J$ & $m_{0}=0.0050$ & $m_{0}=0.0025$ & $m_{0}=0.0005$ & extrapolated value \\
\hline 0 & 1.6950 & $0.219(3)$ & $0.233(2)$ & $0.237(3)$ & $0.241(4)$ \\
\hline-3 & 2.0855 & $0.223(2)$ & $0.233(1)$ & $0.234(2)$ & $0.238(4)$ \\
\hline-5 & 2.1855 & $0.223(2)$ & $0.232(1)$ & $0.235(1)$ & $0.237(2)$ \\
\hline \multicolumn{6}{|c|}{ tricritical point } \\
\hline 1.9655 & 0.610 & $1.054(3)$ & $1.072(4)$ & $1.076(3)$ & $1.080(4)$ \\
\hline
\end{tabular}

TABLE 3. The global persistence exponent $\theta_{g}$ for the best data collapse for the $2 d$ Blume-Capel model with initial magnetization $m_{0}=0$.

\begin{tabular}{lccc}
\hline \hline & & \\
& & critical point & tricritical point \\
$L_{1}$ & & $\left(D / J=0, k_{B} T / J=1.6950\right)$ & $\left(D / J=1.9655, k_{B} T / J=0.610\right)$ \\
$\theta_{g}$ & $0.88(2)$ \\
10 & $L_{2}$ & $0.254(7)$ & $1.08(2)$ \\
20 & 20 & $0.26(2)$ & $1.06(2)$ \\
40 & 40 & $0.24(2)$ & $\theta_{g}$ \\
\hline \hline
\end{tabular}




\section{FIGURE CAPTIONS}

Figure 1: Persistence probability $P(t)$ for the $2 d$ Blume-Capel model for $L=80$ at a critical point with the sharp preparation of the initial magnetization $m_{0}$.

Figure 2: Persistence probability $P(t)$ for the $2 d$ Blume-Capel model for $L=80$ at the tricritical point with the sharp preparation of the initial magnetization $m_{0}$.

Figure 3: Collapse of persistence probability at the tricritical point $D / J=1.9655, k_{B} T / J=0.610$.

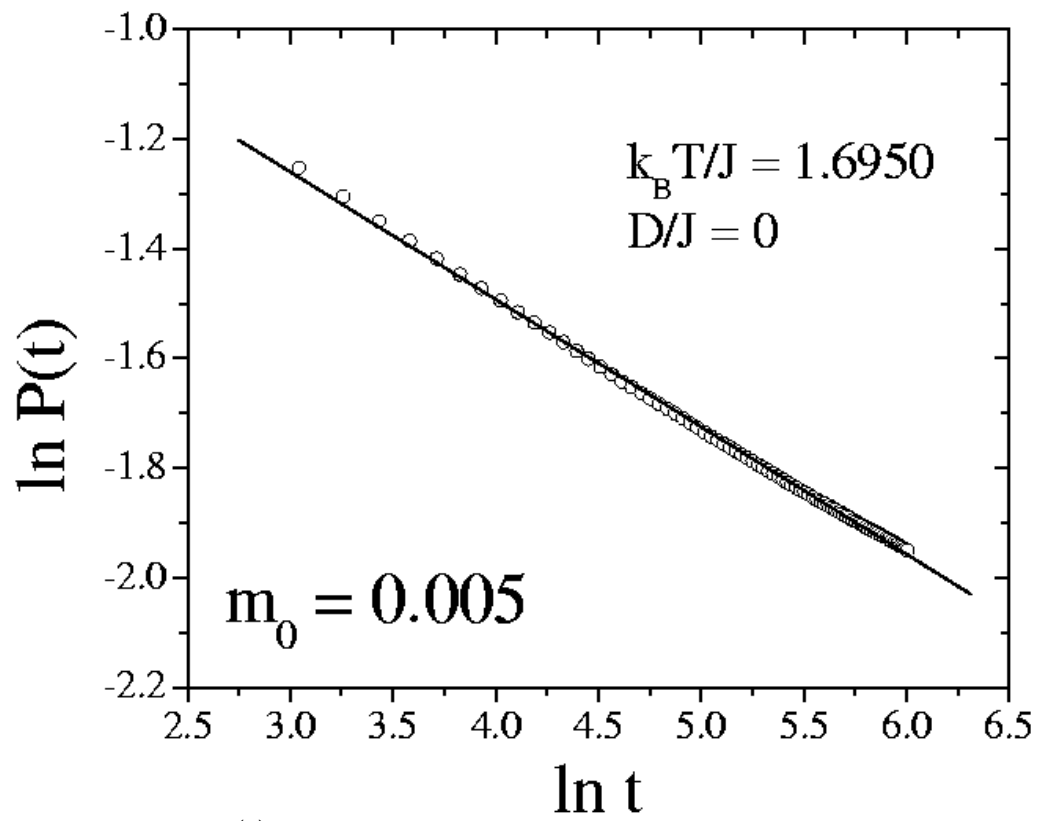

FIG. 1. Persistence probability $P(t)$ for the $2 d$ Blume-Capel model for $L=80$ at a critical point with the sharp preparation of the initial magnetization $m_{0}$. 


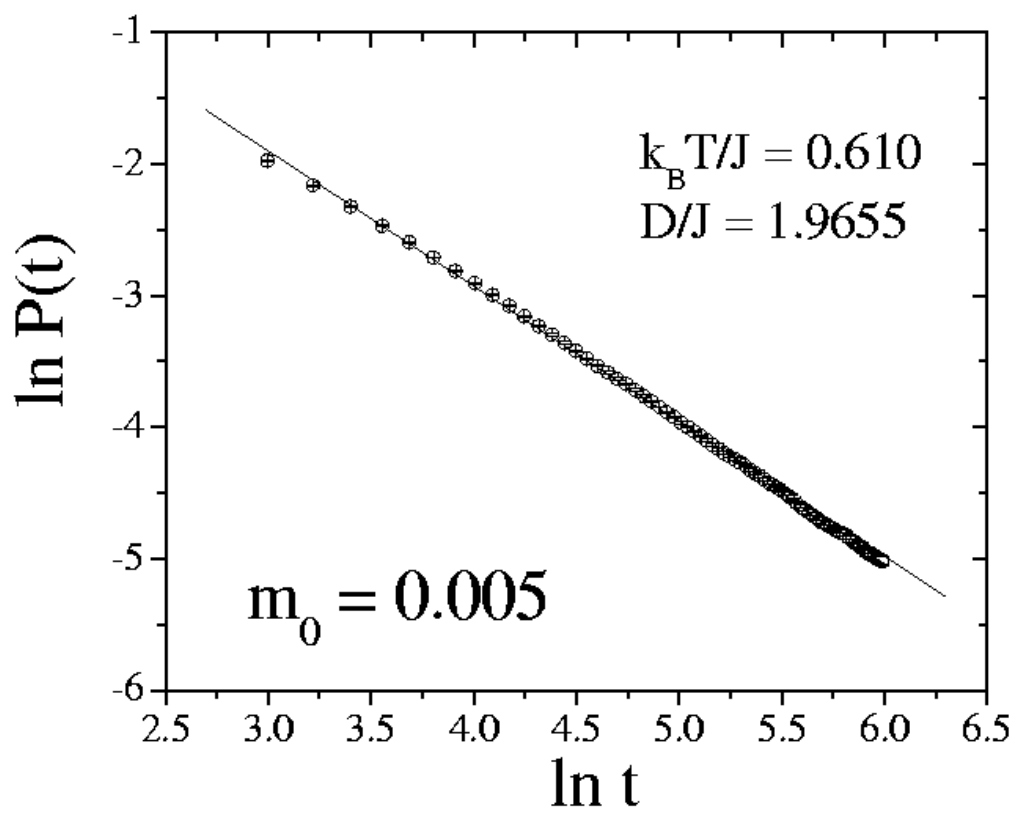

FIG. 2. Persistence probability $P(t)$ for the $2 d$ Blume-Capel model for $L=80$ at the tricritical point with the sharp preparation of the initial magnetization $m_{0}$.

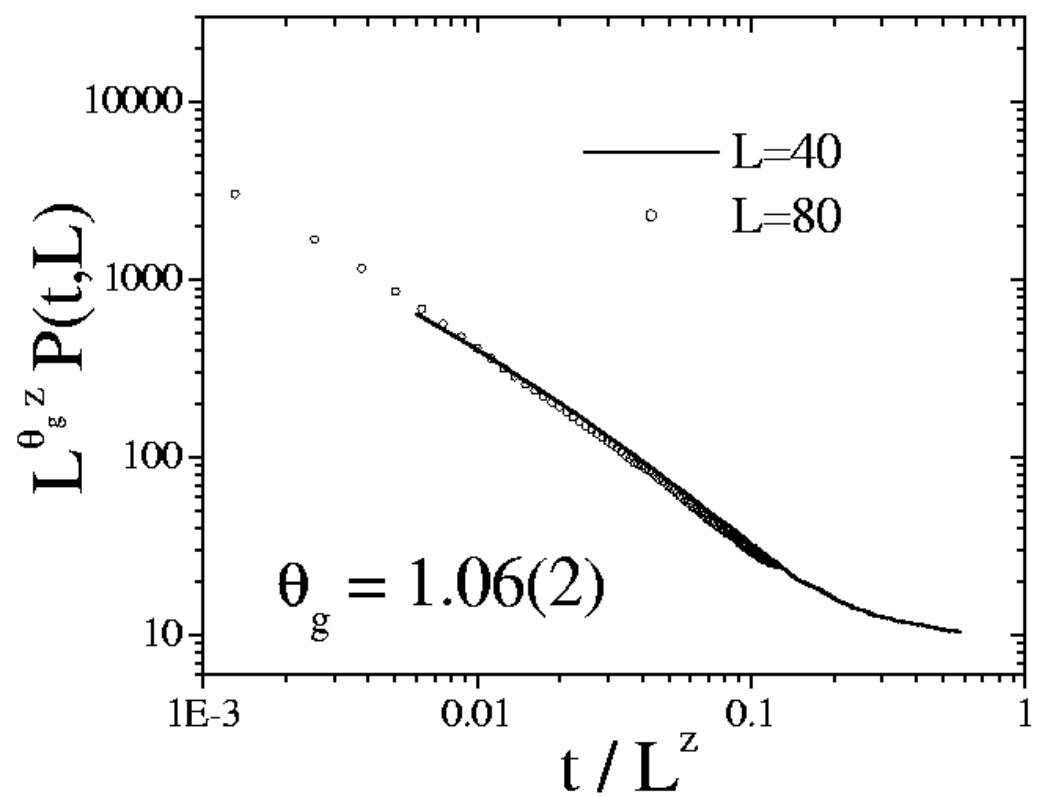

FIG. 3. Collapse of persistence probability at the tricritical point $D / J=1.9655, k_{B} T / J=0.610$. 